To the Editors:

\title{
Surgical closure of ventricular septal defects in Sri Lanka
}

Ventricular septal defects (VSDs) are the commonest congenital heart defects (CHDs) and account for 20-25\% of CHDs [1]. Since 1978, when the repair of four VSDs in the then General Hospital, Colombo was described [2], there has been no documentation of the situation regarding VSD repairs in Sri Lanka. The following study represents our recent experience in surgical closure of VSDs.

A retrospective descriptive study of 128 consecutive patients who underwent VSD repair in the Cardiothoracic Unit 1 of the National Hospital of Sri Lanka from February 1997 to February 2004 was done. The inclusion criterion was the presence of a VSD as the primary lesion. Acquired VSDs were excluded.

Information was obtained from the bed head tickets, operation notes, perfusion records and mortality records. As clinical details of 11 patients could not be retrieved, the remaining 117 records were analysed. The patients' characteristics are shown in Table 1.

All patients underwent median sternotomy and cardiopulmonary bypass at $32^{\circ} \mathrm{c}$. Analysis of the VSD sizes showed that $80 \%$ of the VSDs were large, $11 \%$ medium and $9 \%$ small. Only one patient had multiple VSDs. All VSDs were closed with $0.6 \mathrm{~mm}$ Gore-tex patches. The early outcome is summarised in Table 2.

The optimal management of VSDs requires careful consideration of its natural history. A proportion of VSDs

Table 1. Patients' characteristics

\begin{tabular}{lr}
\hline Characteristic & No. of patients \\
\hline Age & \\
Mean & 8.2 (years) \\
Range & 5 months-37 years \\
Weight & \\
Mean & $8.7(\mathrm{~kg})$ \\
Range & $3-60(\mathrm{~kg})$ \\
Sex & \\
Male & $70(54.68 \%)$ \\
Female & $58(45.32 \%)$ \\
Clinical features & \\
Asymptomatic & $13(11.1 \%)$ \\
Dyspnoea & $69(59 \%)$ \\
Chest pain & $9(7.7 \%)$ \\
Palpitations & $5(4.3 \%)$ \\
Systolic murmur & $117(100 \%)$ \\
Complications & \\
Recurrent respiratory tract infection & $58(49.5 \%)$ \\
Pulmonary hypertension & $58(49.5 \%)$ \\
Heart failure & $42(35.9 \%)$ \\
Failure to thrive & $25(21.4 \%)$ \\
Cyanotic episodes & $5(4.2 \%)$ \\
Right bundle branch block & $1(0.9 \%)$ \\
\hline
\end{tabular}

Vol. 50, No. 2, June 2005
Table 2. Outcome of the Study

\begin{tabular}{lc}
\hline Outcome & No. of patients \\
\hline Morbidity & \\
Respiratory tract infection & $8(6.8 \%)$ \\
Reopening for bleeding & $4(3.41 \%)$ \\
Residual defect requiring redo closure & $2(11.9 \%)$ \\
Pericardial effusion requiring pericardiostomy & $2(1.5 \%)$ \\
Pleural effusion requiring thoracostomy & $2(1.7 \%)$ \\
Cerebral infarction & $2(1.7 \%)$ \\
Infective endocarditis & $1(0.9 \%)$ \\
Complete heart block & $1(0.9 \%)$ \\
Acute renal failure requiring dialysis & $1(0.9 \%)$ \\
Wound infection & $1(0.9 \%)$ \\
Sinus formation & $1(0.9 \%)$ \\
Total & $\mathbf{2 5}(\mathbf{2 1 . 4 \% )}$ \\
Mortality & \\
Acute heart failure & $4(3.1 \%)$ \\
Primary haemorrhage & $2(1.6 \%)$ \\
Cardiac tamponade and arrest & $1(0.9 \%)$ \\
Total & $\mathbf{7 ( 5 . 5 \% )}$ \\
\hline
\end{tabular}

(25-40\%) are known to close spontaneously, the probability of closure showing an inverse relationship to the age at diagnosis [3]. In those children in whom the VSDs persist, a multitude of complications can occur, as was evident in the patients in our series. When the shunt reverses, repair is contraindicated.

The ideal management of VSDs would be to intervene at the time of detection in infancy. Due to the limited facilities and the large number of patients in the National Hospital of Sri Lanka, the policy adopted in our unit is to follow up neonates and infants without pulmonary hypertension (PHT), until the body weight is adequate. Medical therapy to control heart failure and infective complications is given when indicated during this time. Patients with severe PHT detected by two-dimensional echocardiography undergo right heart cardiac catheterization (RHCC). If they are considered operable, pulmonary artery banding is done as the initial procedure in small infants and early repair in older ones. All other patients are operated on as they present via the waiting list.

When simple VSDs without concomitant cardiac lesions are repaired during infancy before the onset of complications in specialised paediatric cardiac centres in the world, the mortality rate is less than $1 \%$ [4]. In the presence of multiple defects and pulmonary hypertension, however, the mortality rate is higher [5]. As only one patient in our series had multiple defects, PHT could have been important in influencing our mortality rates.

The development of PHT is related to the size and the age of the patients, both of which appear to have played a role in this series. Delay in obtaining treatment could be an important contributing factor for the high occurrence 
of PHT. All the VSDs in this series could have been detected on simple auscultation even at the primary health care level to facilitate an early referral. The incidental diagnosis in our series was, however, only $11.1 \%$. Whether the delay in repair was largely due to late detection, late referral, default by the patient or the long waiting list, was not clear.

We have no data on patients who might have died after being discharged. This is a weakness of the study.

In conclusion, this study has shown that the majority of these patients had complications of the VSD by the time they underwent surgery. We have also identified further areas that require study, notably the cause of delay in obtaining treatment and the long term outcome of surgery. These will be key areas for our future studies.

\section{References}

1. Park MK. Pediatric Cardiology for Practitioners. 2nd ed. St Louise: Mosby-Year Book Inc, 1984.

2. Natkunam R. Scope of surgery in congenital heart disease. Sri Lankan Family Physician 1979; 2: 95-9.

3. Brickner ME, Hillis LD, Lange RA. Congenital heart disease in adults-first two parts. New England Journal of Medicine 2000; 342: 256-63.

4. Stark J, Gallivan S, Lovegrove J, Hamilton JR, Monro JL. Mortality rates after surgery for congenital heart defects in children and surgeons' performance. Lancet 2000; 355: 1004-7.

5. Kouchoukos NT, Blackstone EH, Doty DB, Hanley FL, Karp RB, eds. Kirklin/Barratt-Boyes Cardiac Surgery. 3rd ed. Pennsylvania: Elsevier Science, 2003.

DVT Harischandra, Senior Registrar, EMCA Ekanayake, Senior House Office, and GAC Amarasena, Cardiothoracic Surgeon, National Hospital of Sri Lanka, Colombo, Sri Lanka.

Correspondance: DVTH, e-mail: <tolushah@yahoo.co.uk>. (Competing interests: none declared). 